\title{
Dynamic Oscillations of Coupled Domain Walls
}

\author{
L. O'Brien, * E. R. Lewis, A. Fernández-Pacheco, D. Petit, and R. P. Cowburn \\ Thin Film Magnetism Group, Cavendish Laboratory, University of Cambridge, \\ JJ Thompson Avenue, Cambridge CB3 OHE, United Kingdom
}

\author{
J. Sampaio and D. E. Read \\ Experimental Solid State Group, Department of Physics, Blackett Laboratory, Imperial College London, \\ Prince Consort Road, London SW7 2AZ, United Kingdom
}

(Received 2 July 2011; published 30 April 2012)

\begin{abstract}
In domain wall (DW) excitation experiments, nonlinearity (NL) intrinsic to the DW dynamics is often hard to distinguish from perturbation due to the confining potential or DW distortion. Here we numerically investigate the dynamic oscillations of magnetostatically coupled DWs: a system well understood in the quasistatic limit. NL is observed, even for a harmonic potential, due to the intrinsic DW motion. This behavior is principally dependent on terms normally associated with the DW canonical momentum and is in contrast with a NL restoring potential. This NL is not observable in quasistatic measurements, relatively insensitive to the confining potential, and may be tuned by the nanowire parameters. The shown NLs are present in any DW restoring potential and must be accounted for when probing DW potential landscapes.
\end{abstract}

DOI: 10.1103/PhysRevLett.108.187202

The manipulation and control of domain walls (DWs) in ferromagnetic planar nanowires have a wide range of potential technological applications [1-3]. Both in experiment and theory it is found these DWs display a number of particlelike properties. They have an associated mass [4], display inertia $[5,6]$ and will interact with one another [7-9]. Furthermore they may be confined in well-defined potentials [10], with much research undertaken into investigating their dynamic excitation for both fundamental studies and future applications [11-14].

In this work the dynamic, resonant response of magnetostatically interacting DWs in nanowires to a sinusoidal timevarying magnetic field is investigated numerically. This interaction may be well described using only the Coulomb-like magnetostatic energy between two rigid DWs $[7,15]$. We find that even for an entirely harmonic interaction potential, a complex nonlinear response is found in the dynamic regime. This nonlinear response-found to be due to terms normally associated with the DW momentum-results in well-known nonlinear effects such as downshifting of the resonant frequency, hysteretic bistability, and higher order harmonic generation. As these effects are associated with the DW velocity they may be entirely decoupled from both the amplitude response of the DWs and also the specific confining potential shape. The results obtained are directly applicable to any DW confining potential and as such have immediate implications for any investigations probing the resonant excitation of DWs. They show the complexity in characterizing the energy landscape experienced by a DW and highlight the care that must be taken when investigating their dynamic response.

For the nanowire geometries studied in this work [Permalloy (Py), $<100 \mathrm{~nm}$ wide, $<12 \mathrm{~nm}$ thick], the
PACS numbers: 75.60.Ch, 75.60.Jk, 75.75. $-\mathrm{c}, 85.70 . \mathrm{Kh}$

transverse Néel DW (TDW) is the equilibrium configuration $[16,17]$. A Néel DW has a net divergence of magnetization and may be described using a magnetostatic charge density, defined as $\rho=-\mu_{0} \boldsymbol{\nabla} \cdot \mathbf{M}$. DWs with magnetization diverging inwards [outwards], i.e., head-to-head (HH) [tail-to-tail (TT)] DWs, have a net positive [negative] magnetostatic charge. Figure 1(a) shows an example of the micromagnetic configuration [18] $(\alpha=0.5,4 \times 4 \times$ $4 \mathrm{~nm}^{3}$ cell size) in the system under investigation: A HH and TT TDW magnetostatically coupled in two adjacent identical Py nanowires $\left(M_{s}=800 \mathrm{kA} / \mathrm{m}, A=13 \mathrm{pJ} / \mathrm{m}\right)$. The potential is attractive and so the DWs remain coupled despite a magnetic field applied along the $x$ axis of $H=140$ Oe. Here, $w$ is the nanowire width, $t$ the thickness, and $d$ the perpendicular separation (in the figure, $w=50 \mathrm{~nm}, t=8 \mathrm{~nm})$. The central position of DW " $n$ " along the $x$ axis is denoted $x_{n}$, with $n=1$ for the HH DW and $n=2$ for the TT DW.

If the DWs remain rigid, the magnetostatic energy landscape of this interaction, $U_{\text {Int }}$, is well defined and predominantly depends upon DW separation in the $x$ direction, $r$ (where $r=x_{2}-x_{1}$ ) [7]. For a given $d, U_{\text {Int }}$ may be obtained from quasistatic micromagnetic simulations. For DWs which do not appreciably distort, $U_{\text {Int }}$ may be fitted with a harmonic potential, $U_{\text {Int }}=K r^{2}+U_{0}$, up to the point of DW depinning $r=r_{D}$. Figure 1(b) shows the variation of $K$, effectively the "spring constant" between DWs, in this fitting as a function of $w / d$ for $t=8 \mathrm{~nm}$. The effect of thickness variation is not shown in the plot; however, for this range of $w$ and $d$ it is found that $K \propto$ $t^{2}$, as would be expected when considering the scaling of total magnetostatic charge with $t$. In the plot shown, values of constant $d$ are connected by solid black lines. Points of 


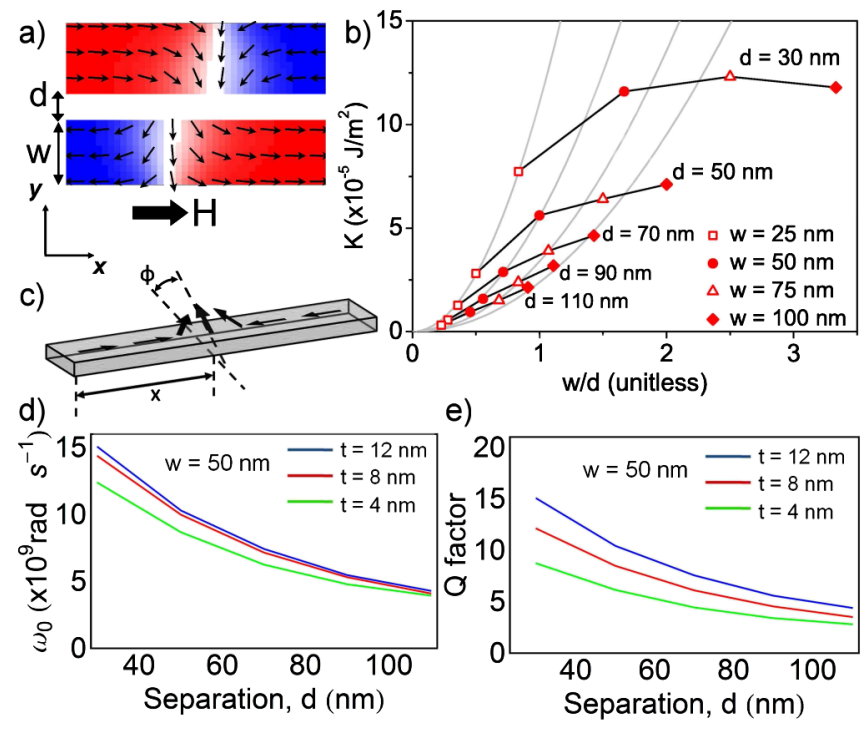

FIG. 1 (color online). (a) Micromagnetic simulation of two magnetostatically coupled HH and TT DWs in parallel nanowires (see text for details). (b) DW-DW interaction coupling strength $K$ as a function of width $w$ and wire separation $d$. (c) Parameters of the DW 1D model: uniform cant angle $\phi$ and central position $x$. (d) Resonant frequency of DW-DW interaction as a function of separation, $d$, and thickness $t(w=50 \mathrm{~nm})$. (e) $Q$ factor of resonance as a function of $d$ and $t(w=50 \mathrm{~nm})$.

constant $w$ are indicated by symbols. The grey curves show the far-field $(w / d<1) K \propto w^{2}$ dependence.

To investigate the dynamic response of the DWs in their mutual potential a 1D model for DW motion is employed, as is commonly used in many DW dynamics problems [11-14]. Figure 1(c) shows the parameters of the system investigated: $\phi$ is the angle $\mathbf{M}$ makes with the $x y$ plane, and $x$ represents the DW central position. Following the treatment of Refs. [19-21], the equations governing the motion of a $1 \mathrm{D}$ rigid DW $n$ are given by:

$$
\begin{aligned}
Q_{n} \frac{\dot{x}_{n}}{\Delta_{n}}-\alpha \dot{\phi}_{n} & =\frac{\gamma_{0}}{\mu_{0} M_{s}}\left[K_{s} \sin \left(2 \phi_{n}\right)\right] \\
Q_{n} \dot{\phi}_{n}+\frac{\alpha}{\Delta_{n}} \dot{x}_{n} & =\frac{\gamma_{0}}{2 \mu_{0} M_{s} S}\left[-\frac{\partial U_{n}}{\partial x_{n}}\right] \\
\dot{\Delta}_{n} & =\frac{12 \gamma_{0}}{\alpha \mu_{0} M_{s} \pi^{2}}\left[\frac{A}{\Delta_{n}}-\Delta_{n}\left(K_{0}+K_{s} \sin ^{2} \phi_{n}\right)\right] .
\end{aligned}
$$

In these equations, $\Delta$ is the DW width parameter, $M_{s}$ the nanowire saturation magnetization, $\alpha$ the damping parameter, $A$ the exchange stiffness, $K_{0}$ the in-plane anisotropy ( $y$ direction) and $K_{s}$ the out-of-plane anisotropy ( $z$ direction). $Q_{n}$ is determined by the net magnetostatic charge of the TDW, +1 for $\mathrm{HH}$ and -1 for TT. $U_{n}$ denotes the total energy of DW $n$ and is given by the sum of the DW internal energy $\left(U_{0}\right)$, Zeeman energy, and interaction energy with any neighboring DWs: $U_{n}=U_{0}-$ $Q_{n} \mu_{0} M_{s} S H x_{n}+U_{\text {Int }} . S=w t$ is the nanowire cross sectional area. The relaxation of $\Delta$ from Eq. (1c) is associated with a "hard mode" that typically occurs on time scales much shorter than DW motion [20,22]. Thus we assume $\Delta$ is given by $\Delta_{n}=\Delta_{0} \sqrt{1 /\left(1+\left(K_{s} / K_{0}\right) \sin ^{2} \phi_{n}\right)}$ throughout excitation of the DWs. An infinite harmonic potential well is assumed to avoid the complications of depinning in characterizing the DW excitation. Under a sinusoidal driving field $H$ and in the limit of small excitation, with $\Delta_{n}=\Delta_{0}(\phi=0)=\sqrt{A / K_{0}}$ and $\sin (2 \phi)=2 \phi$, an analytical solution is obtained, as used previously to study DW excitations $[23,24]$. Using the reduced positioncoordinate $r$ and Eq. (1) we reach a solution similar to that of a simple damped harmonic oscillator (DHO) with natural frequency, $\omega_{0}$, and damping, $\Gamma$, given by:

$$
\begin{gathered}
\omega_{0}=\sqrt{\frac{K}{m_{D}^{*}}}=\sqrt{\frac{4 \Delta \gamma^{2} K_{s} K}{\left(1+\alpha^{2}\right) S M_{s}^{2}}} \\
\Gamma=\left[a_{0}+\frac{\alpha M_{s} K}{\gamma K_{s}}\right] \frac{1}{m_{D}^{*}} \\
m_{D}^{*}=\frac{m_{D}}{2}=\frac{\left(1+\alpha^{2}\right) M_{s}{ }^{2} S}{2 \gamma^{2} \Delta K_{s}} \\
a_{0}=\frac{\alpha S M_{s}}{\gamma \Delta} .
\end{gathered}
$$

In this case $m_{D}^{*}$ is the reduced DW Döring mass [25] and $a_{0} \dot{r}$ the intrinsic damping of a single DW.

The dependence of $\omega_{0}$ and the $Q$ factor of the resonance $\left(\omega_{0} / \Gamma\right)$ on the geometrical parameters of the system are illustrated in Figs. 1(d) and 1(e) $(\alpha=0.01)$. The interaction strength is taken from the empirical values of $K$ found previously [see Fig. 1(c)] while $K_{s}$ and $K_{0}$ are taken from analytical approximations [26]. Displayed are the variations with respect to $d$ for $w=50 \mathrm{~nm}$. For all values of $t$ a monotonic decrease in both $\omega_{0}$ and the $Q$ factor is found for increasing $d$. As would be expected, this arises solely from the $\sqrt{K}$ contribution in $\omega_{0}$. Despite the strong dependence of most parameters $\left(K_{s}, K_{0}, S, K\right.$ and $\left.\Delta\right)$ on both $t$ and $w$, only a weak dependence of $\omega_{0}$ and the $Q$ factor on $t$ is observed and almost no dependence on $w$ is found.

From this linearized, analytical solution to the 1D model we find a low-amplitude sinusoidal magnetic field will excite a mode where the two DWs move in opposite directions analogously to a coupled DHO. The DW cant angle $\phi_{n}$ is often associated with a canonical momentum term, $m_{D} \dot{x}_{n}$, at low $\alpha$ or at constant DW velocity [cf. Eq. (1a)]. It would be expected therefore that the DHO analogy would continue to hold, with the momentum represented in the response of $\phi$. However, the presence of the $Q_{n}$ factor breaks the symmetry between the $\mathrm{HH}$ and TT oscillating DWs. As a consequence, although the DWs move in opposite directions in $x$ under a driving 
magnetic field, they cant in the same direction at all times (i.e., $\phi_{1}=\phi_{2}$ ).

Having investigated the low-drive field regime, we now consider the behavior of the DWs during larger $\phi, \Delta$, and $r$ excursions. The linear solution presented is not appropriate for this regime; therefore, Eq. (1) is solved numerically using a Runge-Kutta order 5 algorithm. Unless otherwise stated, the parameters $w=50 \mathrm{~nm}, t=8 \mathrm{~nm}, d=30 \mathrm{~nm}$, $\Delta_{0}=21 \mathrm{~nm}, K_{s}=247 \mathrm{~kJ} / \mathrm{m}^{3}$, and $\alpha=0.01$ are used. $\Delta_{0}$ and $K_{s}$ are typically estimated from the geometry of the nanowire (which for our geometry would give $\Delta_{0}=14 \mathrm{~nm}$, $K_{s}=265 \mathrm{~kJ} \mathrm{~m}^{-3}$ ); however, different values are used in this work to match the low-amplitude response in micromagnetic simulations (see simulation details later in text).

Figure 2(a) shows the effect of increasing driving field amplitude, $H$, on response amplitude, $r_{\max }$. It can immediately be seen as $H$ increases downshifting, foldover of the peak and bistability (when $H$ exceeds $\sim 15 \mathrm{Oe}$ ) appear, as is often found in weakly nonlinear systems $[27,28]$ and magnetic oscillators $[29,30]$. Irregular, discontinuous jumps are made between the two states of high (out-of-phase with field) and low (in-phase with field) amplitude response within this region. Shown are the boundaries to this hysteretic region averaged over 10 frequency sweeps (error in boundary is $\sim \mathrm{MHz}$ ). As the quasistatic restoring potential of the DWs remains completely harmonic in $r$, the origin of this asymmetry is solely due to terms associated with the $\phi$ response: both the nonlinear demagnetizing $\left(K_{s}\right)$ term in Eq. (1a), and the $\phi$ dependence of $\Delta$ in Eq. (1c). The $K_{s}$ term in Eq. (1a) has many parallels with a nonlinear demagnetizing term in ferromagnetic resonance experiments, where it is found to predominantly cause a foldover response similar to that of a nonlinear restoring potential [31]. However, in the case of DW excitation, the $\phi$ term is better related to the DW momentum due to the equations of motion governing the DW response. As such any nonlinearities are solely dynamic effects (if $\dot{r} \rightarrow 0, \phi \rightarrow 0$ ) and therefore will not cause the same behavior as a nonlinear potential in $r$, nor will they be observable in quasistatic measurements. By linearizing the $\phi$ terms in (1), while allowing $\Delta$ to vary, we may identify the dominant term causing nonlinearity. It is found the onset of nonlinearity occurs for larger $\phi_{\max }$ and $r_{\max }$ when $\Delta$ is the only source of nonlinearity, indicating the $K_{s}$ term in Eq. (1a) is the dominant mechanism in this DW-DW system.

Figure 2(b) shows the change in $r_{\max }$ and maximum cant angle $\phi_{\max }$ as a function of $f$ (increasing in drive frequency) for various $K$ and $\alpha$ values $(H=0.5 \mathrm{Oe})$. Indicated by grey dashed lines are $r_{\max } \propto 1 / f$ dependence (in the linear limit $r_{\max } \propto 1 / f_{0}$ and $f_{0} \propto \sqrt{K}$ ). It can be seen the degree of nonlinearity cannot be directly attributed to the response amplitude, $r_{\max }$ : With $K=$ $20 \times 10^{-5} \mathrm{~J} / \mathrm{m}^{-2}, \alpha=0.001$ the resonant peak is highly asymmetric despite having a lower amplitude than the case of $K=1 \times 10^{-5} \mathrm{~J} / \mathrm{m}^{-2}, \alpha=0.01$. Were the nonlinear a)

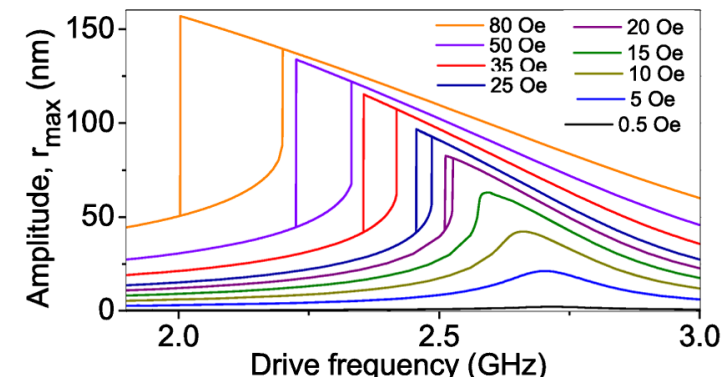

b)
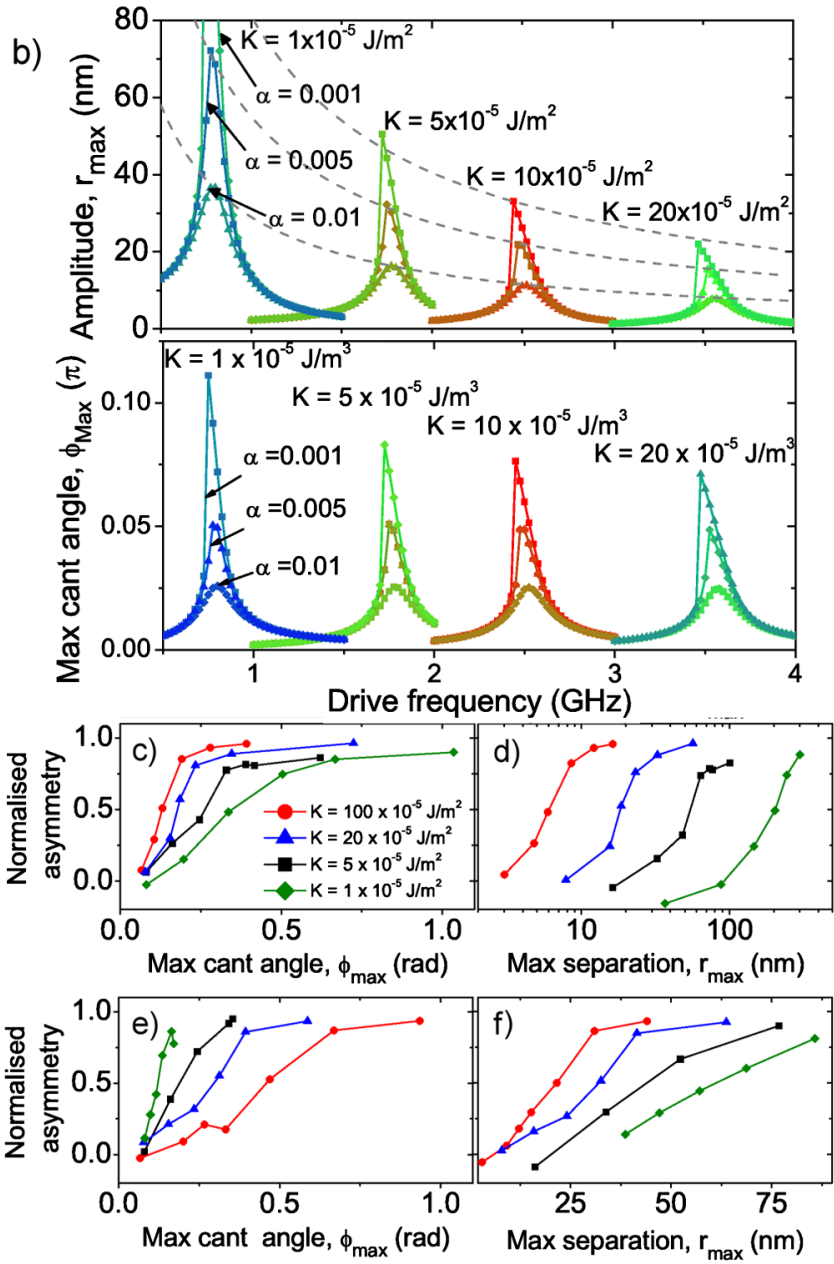

FIG. 2 (color online). (a) Numerically solved, nonlinear amplitude response as a function of magnetic field frequency and amplitude. (b) Amplitude and cant angle response for $K=1$ to $20 \mathrm{~J} / \mathrm{m}^{2}$ and $\alpha=0.01$ to 0.001 (as indicated). Grey dashed lines indicate $\alpha \propto 1 / f_{0}$ dependence. Normalized degree of asymmetry in response for: (c) $\phi$ and (d) $r$ response using full equations of motion [Eq. (1)], and (e) $\phi$ and (f) $r$ response for linearized Eq. (1) with nonlinear restoring potential $U_{\text {int }}^{\prime}\left(\epsilon=-1 \times 10^{13} \mathrm{~m}^{2}\right)$.

behavior due to a nonlinear restoring potential, e.g., replacing $U_{\text {Int }}=K r^{2}$ with $U_{\text {Int }}^{\prime}=K r^{2}\left(1+\epsilon r^{2}\right)$, it would be expected that the degree of nonlinearity be directly determined by $r_{\max }$.

The difference between the observed response and that caused by a nonlinear restoring potential is readily 
observed in Figs. 2(c)-2(f). Plotted is the normalized asymmetry of the resonance peak (taken as the normalized difference between the integrated area on either side of the peak) as a function of $\phi_{\max }$ and $r_{\max }$, for various $K$ values. Figures 2(c) and 2(d) are for the full equations of motion [Eq. (1)], while Figs. 2(e) and 2(f) are for linearized Eq. (1) $\left[\Delta=\Delta_{0}, \sin (2 \phi)=2 \phi\right]$, with the addition of $U_{\text {Int }}^{\prime}(\epsilon=$ $-1 \times 10^{13} \mathrm{~m}^{-2}$ ). For the full equations of motion, the onset of nonlinear behavior is found above a critical value of $\phi_{\max }$ and has a weak inverse dependence on $K$. This dependence arises due to the fact $K$ contributes to the damping of the oscillator, even in the linear regime [c.f. Eq. (3)]: in this regime maximum DW momentum, and so $\phi_{\max }$, is determined solely by the balance of any dissipative $(\Gamma)$ and driving terms $(H)$. The values of $r_{\max }$ at which asymmetry occurs are however highly dependent upon $K$; in the linear limit $\phi_{\max } \sim m_{D} \dot{r} \propto \omega_{0} r_{\max }$, hence $r_{\max } \propto$ $1 / \sqrt{K}$ for approximately constant values of $\phi_{\max }$. Conversely, for a nonlinear restoring potential $U_{\text {Int }}^{\prime}$ a very different $K$ dependence is found. Now asymmetry is determined by a threshold $r_{\max }$, which is inversely dependent upon $K$ (again as $K$ weakly affects $\Gamma$ ). For constant $r_{\max }$, $\phi_{\max }$ scales approximately with $\sqrt{K}$; therefore, we now observe increasing $\phi_{\max }$ for increasing $K$-in strong contrast with the previous case. Identical behavior is observed for $\epsilon= \pm 1 \times 10^{12}$ and $\pm 1 \times 10^{14} \mathrm{~m}^{-2}$.

Given that the degree of linearity in the system is principally determined by the cant angle response, we may use $\phi_{\max }$ to indicate the dependence of this nonlinearity on the system parameters. In the linear limit, with $\phi \sim m_{D} \dot{x}$ and on resonance:

$$
\phi_{\max }=\frac{\mu_{0} H M_{s}}{2 \alpha \Delta}\left[\frac{K}{S}+\frac{K_{s}}{\Delta}\right]^{-1} .
$$

Equation (6) suggests the degree of linearity of the oscillations may be directly determined by the physical parameters of the nanowire, e.g., $\alpha, S$, and $M_{s}$. Furthermore, as in most cases $K_{s} / \Delta \gg K / S$, this nonlinear behavior is largely independent of the external pinning profile (i.e., $K$ ). This suggests the degree of linearity (relatively insensitive to $K$ ) may be decoupled from the amplitude response of the DWs (dependent on $K$ ).

Further to frequency downshifting and bistability, other nonlinear phenomenon may be observed in the response of the system, for example, higher harmonic generation. Figure 3(a) shows the frequency spectrum of the $r$ response, obtained from numerically solving Eq. (1), with drive frequency $f=2.45 \mathrm{GHz} \quad(H=50 \mathrm{Oe})$. From Fig. 2(a), the system is in the nonlinear high-amplitude regime. Clearly seen are components at odd multiples of the fundamental drive frequency, although at significantly reduced powers. These harmonics are found to only be generated around the bistable region and rapidly attenuate as the $\phi$ response decreases.
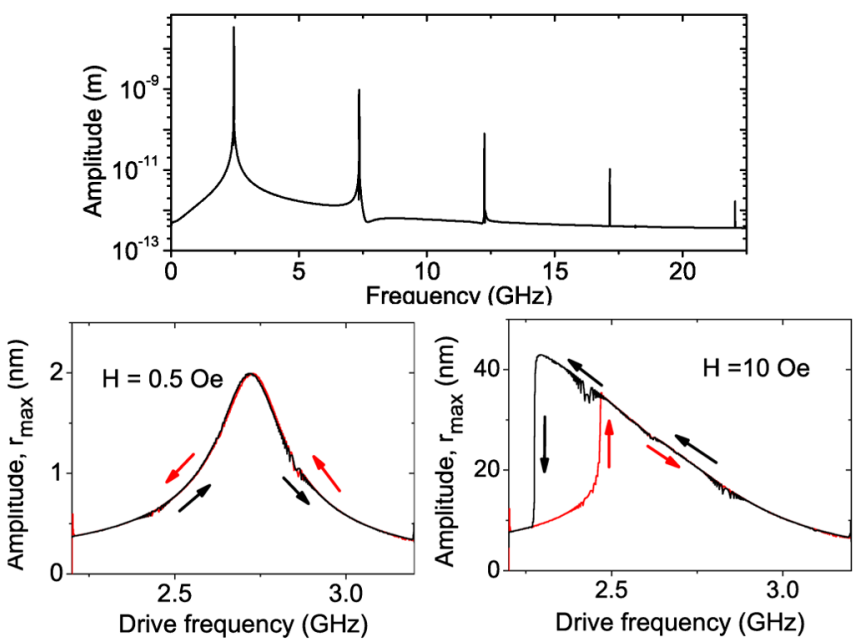

FIG. 3 (color online). (a) Higher harmonics generated in the frequency spectrum of the DW-DW amplitude response $r$ for fixed drive frequency $f=2.45 \mathrm{GHz} \quad(H=50 \mathrm{Oe})$. Micromagnetic simulation positional response for DW-DW interaction for (b) $H=0.5$ Oe and (c) $H=10$ Oe.

We may directly compare this modeled response to that found from full micromagnetic simulations $(\alpha=0.01)$. Figures 3(b) and 3(c) show the obtained DW amplitude response as a function of drive frequency (changing at a rate of $1 \mathrm{MHz} / \mathrm{ns}$ ) for $H=0.5 \mathrm{Oe}$ and $H=10 \mathrm{Oe}$, respectively. As the $1 \mathrm{D}$ model is fitted to match the low-drive response, in the $H=0.5$ Oe case we find excellent agreement between both simulations and the 1D model. At high-drive fields, 1D modeling and simulations show qualitatively similar responses for comparable field strengths, indicating that even in this regime the behavior may still be qualitatively well described by a 1D model. A substantial frequency shift and hysteretic region are observed. Both the shift in $f_{0}(\sim 500 \mathrm{MHz})$ and hysteretic region $(\sim 200 \mathrm{MHz})$ are however found to be much larger than those observed in 1D modeling. This discrepancy may be due to additional anharmonicity in the potential observed by the DWs but, as mentioned, this cannot easily be separated from the intrinsic nonlinear response of the DWs themselves.

In conclusion, the dynamic, resonant response of magnetostatically interacting TDWs has been numerically investigated. This system has previously been well characterized in the quasistatic regime and instrinsic nonlinearity may be isolated from those due to the external DW potential. In the dynamic regime a nonlinear response is observed, even in the case of a harmonic potential, due to the intrinsic motion of the DWs. This behavior is principally dependent on terms normally associated with the DW canonical momentum (however, only weakly dependent upon excitation amplitude) and may be contrasted with a nonlinear restoring potential. This nonlinearity is not observable in quasistatic measurements, is relatively insensitive to the confining potential, and may be directly tuned by 
the nanowire material and geometrical parameters. Through the combination of dynamic and quasistatic measurements of a potential it should be experimentally possible to isolate the contribution of these intrinsic nonlinearities to the motion of a DW. The resulting nonlinearity shown will be present in any DW restoring potential and must be accounted for when probing DW potential landscapes.

We thank O. Wessely for fruitful discussions. The work and results reported in this Letter were obtained with research funding from the European Community under the Seventh Framework Programme Contract No. 247368: 3SPIN.

Note added in proof.-After submission of this work we became aware of a related article [32].

*lao24@cam.ac.uk

[1] G. Vieira, T. Henighan, A. Chen, A. J. Hauser, F. Y. Yang, J. J. Chalmers, and R. Sooryakumar, Phys. Rev. Lett. 103, 128101 (2009).

[2] S. S. P. Parkin, M. Hayashi, and L. Thomas, Science 320, 190 (2008).

[3] D. A. Allwood, Gang Xiong, M. D. Cooke, C. C. Faulkner, D. Atkinson, N. Vernier, and R. P. Cowburn, Science 296, 2003 (2002).

[4] E. Saitoh, H. Miyajima, T. Yamaoka, and G. Tatara, Nature (London) 432, 203 (2004).

[5] J. Rhensius, L. Heyne, D. Backes, S. Krzyk, L. J. Heyderman, L. Joly, F. Nolting, and M. Kläui, Phys. Rev. Lett. 104, 067201 (2010).

[6] L. Thomas, R. Moriya, C. Rettner, and S. S. P. Parkin, Science 330, 1810 (2010).

[7] L. O'Brien, D. Petit, H. T. Zeng, E. R. Lewis, J. Sampaio, A. V. Jausovec, D. E. Read, and R. P. Cowburn, Phys. Rev. Lett. 103, 077206 (2009).

[8] M. Laufenberg et al., Appl. Phys. Lett. 88, 212510 (2006).

[9] R. L. Stamps, A. S. Carriço, and P. E. Wigen, Phys. Rev. B 55, 6473 (1997).

[10] L. O'Brien, D. Petit, E. R. Lewis, R. P. Cowburn, D. E. Read, J. Sampaio, H. T. Zeng, and A.-V. Jausovec, Phys. Rev. Lett. 106, 087204 (2011).

[11] J. H. Franken, R. Lavrijsen, J. T. Kohlhepp, H. J. M. Swagten, and B. Koopmans, Appl. Phys. Lett. 98, 102512 (2011).
[12] R. Moriya, L. Thomas, M. Hayashi, Y. B. Bazaliy, C. Rettner, and S.S.P. Parkin, Nature Phys. 4, 368 (2008).

[13] D. Bedau, M. Kläui, M. T. Hua, S. Krzyk, U. Rüdiger, G. Faini, and L. Vila, Phys. Rev. Lett. 101, 256602 (2008).

[14] A. Bisig, L. Heyne, O. Boulle, and M. Kläui, Appl. Phys. Lett. 95, 162504 (2009).

[15] T. J. Hayward, M. T. Bryan, P. W. Fry, P. M. Fundi1, M. R. J. Gibbs, D. A. Allwood, M.-Y. Im, and P. Fischer, Phys. Rev. B 81, 020410(R) (2010).

[16] R. D. McMichael and M. J. Donahue, IEEE Trans. Magn. 33, 4167 (1997).

[17] Y. Nakatani, A. Thiaville, and J. Miltat, J. Magn. Magn. Mater. 290, 750 (2005).

[18] M. J. Donahue and D. G. Porter, Interagency Report No. NISTIR 6376, 1999.

[19] A.P. Malozemoff and J.C. Slonczewski, Advances in Materials and Device Research (Academic Press, New York, 1979).

[20] A. Thiaville and N. Nakatani, in Spin Dynamics in Confined Magnetic Structures III (Springer, New York, 2006).

[21] N. L. Schryer and L. R. Walker, J. Appl. Phys. 45, 5406 (1974).

[22] O. A. Tretiakov, D. Clarke, G.-W. Chern, Ya. B. Bazaliy, and O. Tchernyshyov, Phys. Rev. Lett. 100, 127204 (2008).

[23] M. Hayashi, L. Thomas, C. Rettner, R. Moriya, and S. S. P. Parkin, Appl. Phys. Lett. 92, 162503 (2008).

[24] L. Bocklage, B. Krüger, R. Eiselt, M. Bolte, P. Fischer, and G. Meier, Phys. Rev. B 78, 180405(R) (2008).

[25] W. Doring, Z. Naturforsch. A 3, 373 (1948).

[26] A. Aharoni, J. Appl. Phys. 83, 3432 (1998).

[27] G. Duffing, Erzwungene Schwingungen bei Veranderlicher Eigenfrequenz und ihre Technische Bedeutung, Sammlung Vieweg Vol. 41/42 (Vieweg \& Sohn, Braunschweig, 1918).

[28] A. H. Nayfeh and D.T. Mook, Non-linear Oscillations (Wiley, New York, 1995).

[29] K. S. Buchanan, M. Grimsditch, F. Y. Fradin, S. D. Bader, and V. Novosad, Phys. Rev. Lett. 99, 267201 (2007).

[30] W. Chen, G. de Loubens, J.-M. L. Beaujour, J. Z. Sun, and A. D. Kent, Appl. Phys. Lett. 95, 172513 (2009).

[31] Y. Gui, A. Wirthmann, N. Mecking, and C.-M. Hu, Phys. Rev. B 80, 060402 (2009).

[32] I. Purnama, M. Chandra Sekhar, S. Goolaup, and W. S. Lew, Appl. Phys. Lett. 99, 152501 (2011). 